\title{
Genetic Heterogeneity May in Part Explain Sex Differences in the Familial Risk for Schizophrenia
}

\author{
Jill M. Goldstein, Stephen V. Faraone, Wei J. Chen, and Ming T. Tsuang
}

\begin{abstract}
The purpose of this study was to attempt, in part, to explain significant sex differences in the familial risk (FMR) for schizophrenia found in previous studies. We hypothesized that, like probands, relatives of male vs. female probands may express different forms or subsyndromal symptoms of schizophrenia, i.e., differential expression of flat affect. Studied were 332 schizophrenic probands defined by Diagnostic and Statistical Manual of Mental Disorders, 3rd ed. (DSM-III), criteria and 725 first-degree relatives from well-known retrospective cohort family studies. Results showed that relatives of male probands were at significantly higher risk for expressing flat affect than relatives of female probands, which did not hold for relatives of normal controls. Logistic regression was used to show that when flat affect was incorporated into the definition of affected among relatives, sex differences in FMR disappeared.
\end{abstract}

Key Words: Schizophrenia, sex differences, familial risk, genetics, flat affect, family studies

BIOL PSYCHIATRY 1995;38:808-813

\section{Introduction}

A number of recent studies have now reported significant sex differences in the familial risk for schizophrenia (Bellodi et al 1986; Shimizu et al 1987; Crow 1988; Goldstein et al 1990a; Wolyniec et al 1992). (For an extensive critical review, see Goldstein 1995). These studies, which include American, British, Italian, and Japanese samples, have shown that relatives of female

From the Deparment of Psychiatry, Harvard Medical School, Harvard Institute of Psychiatric Epidemiology and Genetics, Massachusetts Mental Health Center, and Brockton/West Roxbury VA Medical Center (JMG; SVF; MTT); Pediatric Psychopharmacology Unit, Psychiatry Service, Massachusetts General Hospital, Boston, Mass. (SVF); the Department of Epidemiology, Harvard School of Public Health, Boston, Mass. (MTT); and the National Taiwan University College of Medicine, Institute of Public Health (MTT).

Address reprint requests to Jill M. Goldstein, Ph.D., Department of Psychiatry (116A), Brockton/West Roxbury VA Medical Center, 940 Belmont Street, Brockton, MA 02401.

Received October 3, 1994; revised January 6, 1995 schizophrenics have a significantly higher risk for schizophrenia than relatives of male schizophrenics.

There is some controversy over whether the effect of sex on familial risk applies to all spectrum disorders (i.e., schizoaffective disorder, psychosis not otherwise specified, schizotypal personality disorder) in addition to schizophrenia. One study has reported a significant effect for all spectrum disorders (Bellodi et al 1986). However, this study did not interview relatives, and therefore a spectrum disorder such as schizotypal personality disorder would be underreported in such a study. In contrast, a recent family study (i.e., one in which relatives were personally interviewed), reported that relatives of female patients had a higher risk for the psychotic spectrum disorders, (i.e., schizophrenia, schizophreniform, and schizoaffective disorders) whereas relatives of male patients had a significantly higher risk for schizotypal personality disorder (Goldstein et al 1990a).

Few studies, however, have attempted to explain the 
effect of sex on the familial risk for schizophrenia (Crow 1988; Pulver et al 1990; Goldstein et al 1992). Some of the possible explanatory variables have included age at onset, premorbid history, and symptom expression, since a number of studies have demonstrated significant sex differences in these factors (Gittelman-Klein and Klein 1969; Lewine 1988; Goldstein and Link 1988). That is, compared to women, schizophrenic men have been found, among other things, to have a significantly younger age at onset, poorer premorbid history, and more of the negative symptoms of schizophrenia, in particular, flat affect. One study suggested that age at onset might account for familial risk differences (Pulver et al 1990). However, this was not replicated (Goldstein et al 1992). In fact, Goldstein and colleagues (1992) showed that sex differences in familial risk for schizophrenia were not explained by sex differences in age at onset, premorbid history, season of birth, nor symptom expression. In addition, Crow's (1988) hypothesis of a pseudoautosomal locus for schizophrenia does not explain the higher risk among relatives of female schizophrenics. However, recent work by his group (Crow et al 1994) and DeLisi and colleagues (1994) suggesting a dominant X-Y model of transmission provides a possible explanation for sex differences in familial transmission (see Discussion section).

The study presented here also suggests a genetic explanation that involves possible genetic heterogeneity. If genetically meaningful subtypes of schizophrenia have different sex distributions, relatives of male and female patients might express different forms of schizophrenia. In a recent article, our findings suggested that male and female schizophrenics may be at differential risks for expressing particular forms of schizophrenia, resulting in a difference in prevalence of subtypes (Goldstein et al $1990 \mathrm{~b})$. This was supported by recent work in Britain (Castle et al 1994). Thus, if the finding were valid, perhaps relatives of male and female schizophrenics may also differentially express the gene(s) for the illness. The differential expression may be manifest as spectrum disorders or may be something other than a psychiatric diagnosis.

For example, Tsuang and colleagues (1991) found significantly higher rates of negative symptoms, in particular, flat affect, in otherwise unaffected first degree relatives of a large Veterans Administration (VA) population sample. Given that their sample consisted entirely of men, and that studies have found that schizophrenic men express significantly more flat affect than women (Lewine 1988; Goldstein and Link 1988), we hypothesized that perhaps the abnormal gene(s) for one form of schizophrenia were more likely to be expressed as flat affect among relatives of male schizophrenic probands than among relatives of female probands. In fact, male schizophrenic probands themselves, in the sample on whom we tested this hypothesis, expressed significantly more flat affect than schizophrenic women (Goldstein et al 1990b). A higher family morbidity risk for flat affect among relatives of male schizophrenics would also be consistent with our previous finding in this sample of higher rates of schizotypal personality disorder (SPD) among relatives of male than female probands (Goldstein et al 1990a), since the diagnosis of SPD includes a number of negative symptoms.

\section{Methods}

The data for this study have been described extensively in numerous previous publications, including three of our recent publications on the effect of sex on the familial risk for schizophrenia (Goldstein et al 1990a, 1992, 1993). The data were from the double-blind retrospective cohort family studies conducted in Iowa: the Iowa 500 and non500 studies (Morrison et al 1972; Tsuang et al 1976). Probands were selected from a blind review of index admissions, follow-up records, and interviews of 510 consecutively admitted patients who had a chart diagnosis of schizophrenia, from the Iowa Psychopathic Hospital during 1934-1944. Probands and relatives were then rediagnosed by two expert psychiatrist-diagnosticians using DSM-III criteria (Kendler et al 1985). The criterion limiting age at onset to 45 years was eliminated, as in DSM-III revised (DSM-III-R). Diagnoses of 725 firstdegree relatives were based on a blind, personal structured psychiatric interview $(n=642)$ (Tsuang et al 1980), hospital records obtained from a systematic search of the five Iowa public psychiatric hospitals $(n=55)$, or a combination of interview and records $(n=28)$. Diagnostic procedures and excellent reliability were reported in a previous publication (Kendler et al 1985).

Normal controls $(n=318)$ were sampled from patients undergoing appendectomies and herniorrhaphies from the Iowa general hospital from 1938 to 1948 , and matched by age, sex, and health insurance status, as an indicator of socioeconomic status. All controls were screened for psychiatric symptoms. Control subjects had 1359 siblings and 988 children. For the purpose of the analyses presented here, normal controls were used to examine the rates of flat affect in the relatives of normals for male and female subjects.

Probands included 332 DSM-III diagnosed schizophrenics, $51.5 \%$ men and $48.5 \%$ women. Detailed descriptions of the probands and relatives were provided by Goldstein et al (1989). The sample consisted of a middle to lower middle class, non-Hispanic white population of consecutive admissions to a tertiary care, universityaffiliated facility over a 10 year period. They were 
Table 1. Risk of Flat Affect Among Relatives With No DSM-III Diagnosis

\begin{tabular}{|c|c|c|c|c|c|c|}
\hline \multirow[b]{2}{*}{ Group } & \multicolumn{2}{|c|}{ Relatives of Men } & \multicolumn{2}{|c|}{ Relatives of Women } & \multirow[b]{2}{*}{$\chi^{2}(\mathrm{df}=1)$} & \multirow[b]{2}{*}{$p$} \\
\hline & Sample size & Risk (\%) & Sample size & Risk (\%) & & \\
\hline Schizophrenia & 245 & 2.9 & 291 & 0.7 & 3.8 & 0.05 \\
\hline Control & 246 & 1.6 & 265 & 2.6 & 0.6 & 0.43 \\
\hline
\end{tabular}

primarily in the early stages of disorder; $82 \%$ were first admissions, and were evaluated for up to 42 years. (The median observation period was 32 years.) They had a mean age of 27 years, with men showing onset at a significantly earlier age than women $(24.3 \pm 8.3$ years vs. $27.9 \pm 6.2$ years [Goldstein et al 1989]). Forty-eight percent had completed secondary education, and 32\% had had some college education. The majority had never been married $(70 \%)$ and only $16 \%$ were unemployed at the time of admission.

Of the 332 probands there were 253 for whom clinical information on first-degree relatives was available. The majority $(72 \%)$ of the 725 relatives were siblings, $9 \%$ were parents, and $19 \%$ were children. Forty-five percent were male and $55 \%$ were female.

Symptoms and other covariates of interest in this study were rated by trained interviewers using a structured psychiatric interview developed by Tsuang and colleagues, medical records, and relatives. Reliability and validity of the instrument have been reported in numerous publications (Tsuang et al 1976, 1980; Kendler et al 1987). For the purpose of this study, we were interested in ratings of flat or blunted affect in relatives. The presence or absence of flat or blunted affect was systematically coded at the end of the interview by the trained interviewers. The ratings were based on the observation of the subject during the interview. This procedure is similar to the way in which flat or blunted affect is rated using current instruments. However, we are aware that at the time this study was conducted negative symptoms were not as prominent a concern in schizophrenia research as today. Thus, flat/blunted affect may be underreported in this study. However, we were interested in the differential expression of flat affect among relatives of male vs. female probands, and there is no reason to believe that there was a differential underreporting, if at all, among relatives of male and female probands. Reliability of the behavioral observations, including flat or blunted affected, in the Iowa study was satisfactory (intraclass correlation coefficient of approximately 0.57 ), although reliability was not calculated for individual behavioral items.

First, using odds ratios, we examined the risk for flat affect among otherwise unaffected relatives of male and female probands. A Breslow-Day test (Breslow and Day 1980) of the homogeneity of the odds ratios between the two groups was used to examine the significance of the differences in the risk among male vs. female probands. We were then interested in testing whether we could account for the significant effect of sex on the risk for schizophrenia and/or psychotic spectrum disorders, found in previous work (e.g., Goldstein et al 1990a), by incorporating flat affect in the definition of "being affected" among the relatives, in addition to schizophrenia and/or the psychotic spectrum disorders. That is, if we showed that the effect of sex was not significant when we included flat affect in the definition of the affected phenotype, this would indicate that the effect of sex on schizophrenia alone was explained in part by differences in the expression of flat affect among male vs. female probands. Logistic regression (Cornfield et al 1961) was used to test for the effect of sex on the familial risk for schizophrenia, schizophreniform, schizoaffective disorders, and flat affect. Relatives' sex and an interaction between relatives' and probands' sex were controlled for in the analysis. If the effect of sex was accounted for by a sex-specific expression of the gene(s), one would expect to see a sex effect among the relatives as well as among the probands. That is, all men or all women would be affected, whether they were probands or relatives. We hypothesize a significant sex effect only among the probands. Two-tailed tests of significance were used for all analyses.

\section{Results}

Table 1 presents a comparison of relatives of male and female schizophrenic probands and relatives of male and female controls. It shows the percentage of relatives who did not have any psychiatric diagnosis but who expressed flat affect. Relatives, without any psychiatric diagnosis, of male schizophrenic probands expressed significantly more flat affect than relatives of female probands $(2.9 \%$ vs $.7 \%$, $\chi^{2}=3.8$, df $=1, p=0.05$ ), and this did not hold true for normal controls. The odds ratio for expressing flat affect among otherwise unaffected relatives of male versus female probands was 4.25 (Standard error [s.e.] 0.8, 95\% Coefficient index $[$ c.i.] $=0.39,9.1)$. In contrast, the odds ratio for relatives of male vs female controls was 0.6 (s.e. $0.6,95 \%$ c.i. $=0.23,2.8$ ), indicating less flat affect among relatives of male controls compared to female controls. A Breslow-Day test of the homogeneity of odds ratios 
Table 2. Logistic Regressions Showing Effects of Sex of Proband and Relative on the Familial Risk for Schizophrenia vs. Risk for Schizophrenia or Flat Affect

\begin{tabular}{|c|c|c|c|c|c|c|c|c|}
\hline & \multicolumn{4}{|c|}{ In Relatives: Schizophrenia Alone } & \multicolumn{4}{|c|}{ Schizophrenia or Flat Affect } \\
\hline & beta & s.e. & $\chi^{2}(\mathrm{df}=1)$ & $p$ & beta & s.e. & $\chi^{2}(\mathrm{df}=1)$ & $p$ \\
\hline Proband's sex & 0.88 & 0.44 & 3.9 & 0.048 & 0.27 & 0.35 & 0.59 & 0.44 \\
\hline Relative's sex & -0.13 & 0.39 & 0.12 & 0.73 & -0.09 & 0.34 & 0.08 & 0.78 \\
\hline Intercept & -3.75 & 0.43 & & & -3.06 & 0.33 & & \\
\hline
\end{tabular}

between schizophrenics and controls was significant, indicating that the two odds ratios were significantly different $\left(\chi^{2}=3.9\right.$, df $\left.=1, p=0.048\right)$.

In addition, Table 1 shows that the risk for flat affect did not differ significantly for relatives of male probands, male controls, and female controls. That is, the chi-square comparing $2.9 \%$, the rate among relatives of male probands, with $1.6 \%$, the rate among male controls, was not significantly different. In contrast, the chi-square comparing $2.6 \%$, the rate among relatives of female controls, with $0.7 \%$, the rate among relatives of female probands, was $3.3(\mathrm{df}=1), p=0.07$. This suggests that, among relatives of female probands, there may be a "protective" effect operating against the expression of flat affect.

A series of logistic regressions were then performed to test for differences in the effect of sex on the familial risk for schizophrenia compared to the effect of sex on the risk for schizophrenia or flat affect. Table 2 presents the logistic regressions showing the effects of sex on the risk for schizophrenia alone compared to the risk for schizophrenia or flat affect, controlled for relative's sex. Table 3 presents the actual risks on which the significance levels in Table 2 were based. As presented in our previous publication (Goldstein et al 1990a), the effect of the proband's sex on the risk for schizophrenia alone was significant.

Table 3. Risk of Schizophrenia and Expanded Definition of the Phenotype Among 725 Relatives of Schizophrenia Male vs. Female Probands

\begin{tabular}{|c|c|c|c|c|}
\hline \multirow[b]{3}{*}{ Sex } & \multicolumn{4}{|c|}{ Diagnosis in Relatives } \\
\hline & \multicolumn{2}{|c|}{ Schizophreni $\mathbf{a}^{a}$} & \multicolumn{2}{|c|}{$\begin{array}{l}\text { Schizophrenia or } \\
\text { Flat Affect }\end{array}$} \\
\hline & $n$ & Risk (\%) & $n$ & Risk (\%) \\
\hline \multicolumn{5}{|l|}{ Proband's sex } \\
\hline Male $(n=327)$ & 7 & 2.1 & 14 & 4.3 \\
\hline Female $(n=398)$ & 20 & 5.0 & 22 & 5.5 \\
\hline $\begin{array}{l}\text { Fisher's exact test } \\
\text { (two-tailed) }\end{array}$ & & 0.05 & & 0.50 \\
\hline \multicolumn{5}{|l|}{ Relative's sex } \\
\hline Male $(n=326)$ & 13 & 4.0 & 17 & 5.2 \\
\hline Female $(n=399)$ & 14 & 3.5 & 19 & 4.8 \\
\hline $\begin{array}{l}\text { Fisher's exact test } \\
\text { (two-tailed) }\end{array}$ & & 0.84 & & 0.86 \\
\hline
\end{tabular}

${ }^{a}$ Reported in a previous publication (Goldstein et al 1990a).
However, when we included flat affect in the definition of the affected phenotype, there was no significant effect of proband's sex on the familial risk. There were also no significant interaction effects of proband's and relative's sex on familial risk.

As can be seen in Table 3, the risk for schizophrenia or flat affect among relatives of schizophrenic male probands increased to $4.3 \%$ compared to $2.1 \%$ for schizophrenia alone. Among relatives of female probands, the risk for schizophrenia compared to schizophrenia or flat affect differed only slightly ( $5 \%$ vs. $5.5 \%$ ). When the definition of affected included flat affect or any of the psychotic spectrum disorders (schizophrenia, schizophreniform, and schizoaffective disorders), the results remained the same. That is, including flat affect in the definition of the affected phenotype attenuated the effect of sex on the familial risk.

\section{Discussion}

Results of this study support the hypothesis that relatives of male and female schizophrenic probands may express the abnormal gene(s) for different subtypes of schizophrenia. We are not arguing that there are sex-specific forms of schizophrenia. If that were the case, one would expect to see all females or all males affected with a particular subtype, whether they were probands or relatives. Results show a significant effect for the sex of the proband, but not for the sex of the relative. That is, male and female relatives of male probands expressed significantly more flat affect in the absence of any psychiatric diagnosis than male and female relatives of female probands.

Results in this study are also consistent with the idea that families of female probands are "protected" from the expression of flat affect. That is, the relatives of male probands, of male controls, and of female controls were all not significantly different from each other, even though relatives of male probands expressed the highest prevalence of flat affect compared with the others. Relatives of female probands expressed significantly lower levels of flat affect. Replication of this study is necessary in order to understand whether the explanation of our results is due to "protective" factors among female proband families or to "risk" factors among male proband families. 
Findings in this study are not likely to be spurious, that is, due to a large number of statistical tests. We had a specific hypothesis that was tested, for which the prediction held. Although the coding of flat affect in this study was not psychometrically sophisticated and was satisfactorily reliable, it produced significant results to our specific hypotheses. If the measurement of flat affect was wholly unreliable, one would expect it to be uncorrelated with other variables of interest. In addition, there is no reason to believe that the rating of flat affect would systematically vary with the sex of the proband due to artifacts, such as the sex of the interviewer, given that the relatives were males and females. However, it is important to replicate findings using state-of-the-art instruments of flat affect.

The data in this study are not compatible with a two-threshold multifactorial polygenic model that posits women to have a higher threshold for affection than men. Such a model predicts that the relatives of females are at greater risk for all genetic forms of the disorder (Reich et al 1979), which we do not find. In ours as well as others' previous work, we showed that relatives of women had a higher risk for schizophrenia, schizophreniform, and schizoaffective disorders. Relatives of men expressed more schizotypal personality disorder (Goldstein et al 1990a). Findings in the study reported here showed that relatives of male probands also expressed more flat affect, in the absence of any psychiatric diagnosis. The differential expression of the gene(s) for schizophrenia among relatives of male vs. female probands may lend support to previous work suggesting that schizophrenic men and women themselves are at different risks for expressing particular forms of schizophrenia (Goldstein et al 1990b; Castle et al 1994).

One mechanism for explaining differential expression by sex has recently been suggested by Crow and colleagues (1994) and DeLisi and colleagues (1994). They suggested that the inconsistent results from linkage studies of the telomeric, pseudoautosomal region implicated a schizophrenia gene in a nearby sex-specific region of the $\mathrm{X}$ and $\mathrm{Y}$ chromosomes. Their results showed some support for a dominant $X-Y$ model of transmission in which homologous genes on $\mathrm{X}$ and $\mathrm{Y}$ contributed to the genetic susceptibility to schizophrenia. Although further work is needed before one can make definitive statements about

\section{References}

Bellodi L, Bussoleni C, Scorza-Smeraldi R, Grassi G, Zacchetti L, Smeraldi E (1986): Family study of schizophrenia: Exploratory analysis for relevant factors. Schizophr Bull 12:120-128.

Breslow NE, Day NE (1980): Statistical Methods in Cancer Research. Vol. 1. The Analysis of Case-Control Studies.
$\mathrm{X}-\mathrm{Y}$ transmission in schizophrenia, the model does provide a mechanism for sex differences in the manifestation and familial transmission of schizophrenia. Since homologous genes are not identical, their effects on pathophysiology and phenotypic expression are not necessarily identical. Given that women cannot transmit the $Y$ form of the homologous gene, the model also allows for differences in affected relatives based on the sex of the proband.

A number of previous studies (including some of our own) have suggested that relatives of female schizophrenics may be at a higher genetic risk for the disorder than relatives of male schizophrenics. It is possible that relatives of male schizophrenics may be at lower genetic risk, since, in some studies, male schizophrenics themselves have been found to have had more perinatal complications than women and thus may possibly be sporadic cases (Lewis and Murray 1987). However, the majority of studies, except those of Shimizu et al. (1987) and Goldstein et al. (1990a), that have shown that relatives of female schizophrenics are at significantly higher risk for schizophrenia than relatives of men are family history studies (e.g., Bellodi et al 1986; Wolyniec 1992). Therefore, the identification of less severe genetic forms of the schizophrenia spectrum is underestimated, if at all. In addition, no one has examined in relatives of male vs. female probands the prevalence of other possible genetic expressions of the disorder.

Thus, as the study reported here suggests, one does not necessarily need to look to nongenetic factors to explain the sex differences in familial risk. Rather, men may be at higher risk for a genetic subform of schizophrenia that is less penetrant for psychosis than other forms, yet is expressed in relatives as subsyndromal phenomenology (i.e., negative symptoms). This would not negate the possibility that nongenetic factors may play a greater role in the risk for schizophrenia among men and their relatives than among women and their relatives. However, it suggests that an alternative mechanism may be operating.

The authors thank Deborha L. Catt for manuscript preparation.

This study was supported, in part, by an NIMH grant MH42604 to JMG and MTT, and, in part, by an NIMH Scientist Development Award (K21 MH00976) to JMG.
Lyon: International Agency for Research on Cancer, IRAC Scientific Publication No. 32.

Castle DJ, Sham PC, Wessley S, Murray RM (1994): The subtyping of schizophrenia in men and women: A latent class analysis. Psychol Med 24:41-51. 
Cornfield J, Gordon T, Smith WW (1961): Quantal response curves of rexperimentally uncontrolled variables. Bull Int Stat Inst 38:97-115.

Crow TJ (1988): Sex chromosomes and psychosis. The case for a pseudoautosomal locus. Br J Psychiatry 153:675-683.

Crow TJ, DeLisi LE, Lofthouse R, Poulter M, Lehner T, Bass N, Shah T, Walsh C, Boccio-Smith A, Shields G, Ott J (1994): An examination of linkage of schizophrenia and schizoaffective disorder to the pseudoautosomal region (Xp22.3). $\mathrm{Br} J$ Psychiatry 164:159-164.

DeLisi LE, Devoto M, Lofthouse R, Poulter M, Smith A, Shields G, Bass N, Chen G, Vita A, Morganti C, Ott J, Crow TJ (1994): Search for linkage to schizophrenia on the $X$ and $Y$ chromosomes. Am J Med Genet (Neuropsych Genet) 54:113121.

Gittelman-Klein R, Klein DF (1969): Premorbid asocial adjustment and prognosis in schizophrenia. $J$ Psychiatr Res 7:3553.

Goldstein JM, Link BG (1988): Sex and the expression of schizophrenia. J Psychiatric Res 22:141-155.

Goldstein JM, Tsuang MT, Faraone SV (1989): Sex and schizophrenia: Implications for understanding the nature of the disorder. Psychiatr Res 28:243-253.

Goldstein JM, Faraone SV, Chen WJ, Tolomiczencko G, Tsuang MT (1990a): Sex differences in the familial transmission of schizophrenia. Br J Psychiatry 156:819-826.

Goldstein JM, Santangelo SC, Simpson JC, Tsuang MT (1990b): The role of sex in identifying subtypes of schizophrenia: A latent class analytic approach. Schizophr Bull 16(2):263-275.

Goldstein JM, Faraone SV, Chen WJ, Tsuang MT (1992): Sex and the familial transmission of schizophrenia: Disentangling confounding factors. Schizophr Res 7:135-140.

Goldstein JM, Faraone SV, Chen WJ, Tsuang MT (1993): The role of sex in understanding the transmission of schizoaffective disorder. Br J Psychiatry 163:763-768.

Goldstein JM (1995): Sex and the familial transmission of schizophrenia: A critical review. In Seeman MV (ed), Sex and Psychopathology. Washington, DC: American Psychiatric Association Press.

Kendler KS, Gruenberg AM, Tsuang MT (1985): Psychiatric illness in first-degree relatives of schizophrenic and surgical control patients. Arch Gen Psychiatry 42:770-779.

Kendler K, Tsuang M, Hays P (1987): Age at onset in schizophrenia: A familial perspective. Arch Gen Psychiatry 44:881890.

Lewine RRJ (1988): Sex and schizophrenia. In Tsuang MT, Simpson JC (eds), Handbook of Schizophrenia, Volume 3. Amsterdam: Elsevier, pp 379-397.

Lewis SW, Murray RM (1987): Obstetric complications, neurodevelopmental deviance and schizophrenia. $J$ Psychiatr Res 21:413-421.

Morrison J, Clancy J, Crowe R, et al (1972): The Iowa 500: I. Diagnostic validity in mania, depression, and schizophrenia. Arch Gen Psychiatry 27:457-461.

Pulver AE, Brown CH, Wolyniec P, McGrath J, Tam D, Adler L, Carpenter WT, Childs B (1990): Schizophrenia: Age at onset, sex, and familial risk. Acta Psychiatr Scand 82:344-351.

Reich T, Rice J, Cloninger CR, Wette R, James JW (1979): The use of multiple thresholds and segregation analysis in analyzing the phenotypic heterogeneity of multifactorial traits. Ann Hum Genet 42:371-389.

Shimizu A, Masayoshi $\mathbf{K}$, Yamaguchi $\mathbf{N}$, Torii $\mathbf{H}$, Isake $\mathbf{K}$ (1987): Morbidity risk of schizophrenia to parents and siblings of schizophrenic patients. Jpn J Psychiatry Neurol 41(1):65-70.

Tsuang MT, Dempsey T, Rauscher F (1976): A study of atypical schizophreia: Comparison with schizophrenia and affective disorder by sex, age of admission, precipitant outcome, and family history. Arch Gen Psychiatry 33:1157-1160.

Tsuang MT, Gilbertson MW, Faraone SV (1991): Genetic transmission of negative and positive symptoms in the biological relatives of schizophrenics. In Marneros A, Andreasen NC, Tsuang MT (eds), Negative Versus Positive Schizophrenia. Berlin/Heidelberg: Springer-Verlag, pp 265-291.

Tsuang MT, Woolson RF, Simpson JC (1980): The Iowa Structured Psychiatric Interview: Rationale, reliability and validity. Acta Psychiatr Scand Suppl. 283:1-38.

Wolyniec PS, Pulver AE, McGrath JA, Tam D (1992): Schizophrenia: Sex and familial risk. $J$ Psychiatr Res 26:17-22. 